\title{
Research on fuzzy PID attitude controller for Multi-rotor UAV Shuo Gao ${ }^{1, \text { a) }}$, Qi Wang ${ }^{2, \text { b) }}$, Wen Jiang ${ }^{2, c)}$ and Yan Liu' ${ }^{2, d)}$
}

${ }^{1}$ School of Aircraft Eengineering, Nanchang Hangkong University, Nanchang 330000, China.

${ }^{2}$ School of Aircraft Eengineering, Nanchang Hangkong University, Nanchang 330000, China.

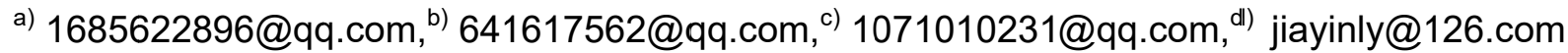

Keywords: Fuzzy PID, Attitude controller, Multi-rotor, UAV

\begin{abstract}
Multi-rotor UAV attitude system has many characteristics such as multi-input multioutput, strong coupling, fast time-varying and non-linearity. Thus, for multi rotor UAV attitude control system, the classic PID has some shortcomings. In this paper, the fuzzy PID controller is established by using fuzzy control and classical PID. The on-line self-tuning of PID parameters is realized. The simulation results show that compared with the classical PID, the fuzzy PID controller has the advantages of high control precision, stronger anti-interference ability and stronger robustness, and is more suitable for multi-rotor UAV attitude control system.
\end{abstract}

\section{INTRODUCTION}

The attitude control of multi rotor UAV has the characteristics of multi-input multi-output, strong coupling, fast time-varying and non-linearity [1]. The classical PID control structure is simple and adaptable, and it is the most widely used control method. The control quality is not sensitive to the change of the controlled object, so it is very suitable for industrial production. Only three parameters $\mathrm{Kp}, \mathrm{Ki}, \mathrm{Kd}$ is needed to be adjusted then you can achieve the desired control effect. In the face of multi rotor UAV attitude control, the classical PID can not meet the requirements of this kind of control. A set of fixed parameters of the classic PID can not be adjusted in time, according to the change of the system, which leads to the decline or even failure of the control quality. If the parameters of the PID controller can be adjusted in time, according to the change of the system, it can improve the applicability of the PID control to the complex system with timevarying and nonlinear dynamics. In this paper, the fuzzy control is used to improve the PID control, the fuzzy controller is used to adjust the PID parameters on-line, and the fuzzy PID controller is established and applied to the attitude control of the multi rotor UAV.

\section{ESTABLISHMENT OF FUZZY PID CONTROLLER}

Fuzzy PID system block diagram is shown in figure 1.

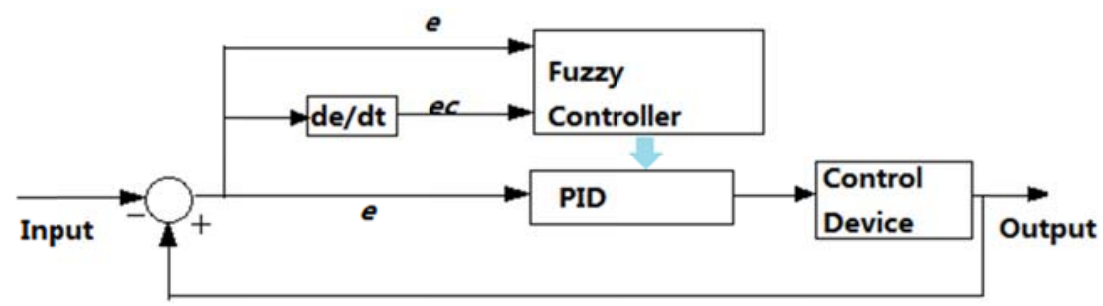

FIGURE 1. Structure of fuzzy PID controller.

The fuzzy controller is composed of database, rule base, fuzzy controller, fuzzy inference engine and fuzzy controller. The fuzzy control system is used as the controller to be a fuzzy controller [2]. In order to improve the speed of the response and reduce the computation, in this paper, the Mamdani type fuzzy controller is chosen. The principle of the Mamdani type fuzzy controller is to express the experience gained through the conditional statement in the real life. The production of inference rule table is completed, which greatly reduces the amount of computation online and reduces the computing power requirements of the hardware platform. This article is used for a long 
time in the field of industrial control PID artificial summary of the parameters of the adjustment experience [3]. The principle of fuzzy controller [4] is shown in figure 2.

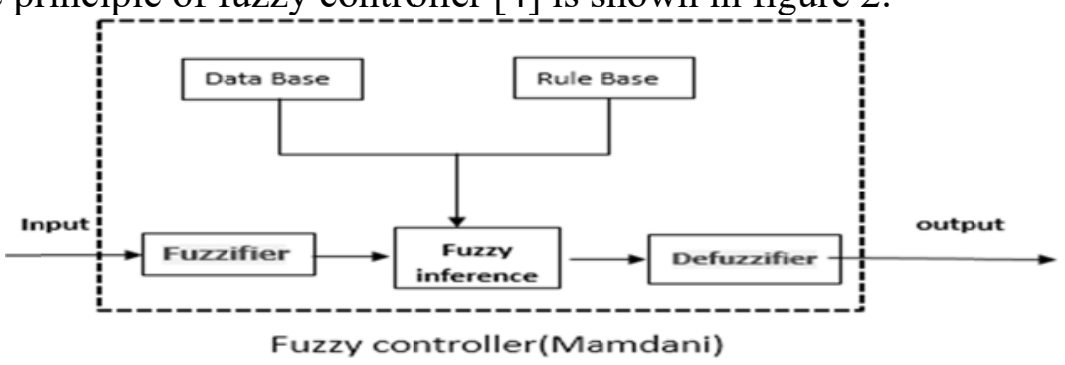

FIGURE 2. Schematic diagram of Mamdani type fuzzy controller.

Select the appropriate data and rules from the database and rule base. Make the rule table according to the dynamic changes of the system and the parameters of the artificial summary [6]. The fuzzy control rule table is shown in table 1. Then the input signal E and EC are transformed into a natural language which can be identified by fuzzy controller. Finally, the fuzzy inference machine is used to process the data. Tuning of PID parameters is complete.

\section{SIMULATION AND ANALYSIS}

In this paper, the classical PID and fuzzy PID in a constant system step response curve of the simulation, dual control system simulation diagram is shown in Figure 3.A pulse strong interference signal with a magnitude of 1 is superimposed on the fifth second, it is shown in figure 4 .

$$
\mathrm{G}=\frac{20}{1.5 S^{2}+4 S+1}
$$

References are cited in the text just by square brackets [1]. Two or more references at a time may be put in one set of brackets $[3,4]$. The references are to be numbered in the order in which they are cited in the text and are to be listed at the end of the contribution under heading references, see our example below.



FIGURE. 3 Simulation diagram of control system.



FIGURE. 4 Comparison curve of step response.

It can be seen from Figure 4 that the delay and overshoot of fuzzy PID are better than the 
classical PID.

TABLE 1. Fuzzy control rule table.

\begin{tabular}{|c|c|c|c|c|c|c|c|}
\hline \multicolumn{8}{|c|}{ Rule table of fuzzypid control of $\Delta \mathrm{kp}$} \\
\hline elec & NB & NM & NS & $\mathrm{ZO}$ & PS & PM & PB \\
\hline NB & PB & PB & PM & PM & PS & $\mathrm{ZO}$ & $\mathrm{ZO}$ \\
\hline NM & PB & $\mathrm{PB}$ & PM & PS & PS & $\mathrm{ZO}$ & NS \\
\hline NS & PM & PM & PM & NS & $\mathrm{ZO}$ & NS & NS \\
\hline $\mathrm{ZO}$ & PM & PM & PS & $\mathrm{ZO}$ & NS & NM & NM \\
\hline PS & PS & PS & $\mathrm{ZO}$ & NS & NS & NM & NM \\
\hline PM & PS & $\mathrm{ZO}$ & NS & NM & NM & NM & NB \\
\hline PB & $\mathrm{ZO}$ & $\mathrm{ZO}$ & NM & $\mathrm{NM}$ & NM & NB & NB \\
\hline \multicolumn{8}{|c|}{ Rule table of fuzzypid control of $\Delta \mathrm{ki}$} \\
\hline elec & NB & NM & NS & $\mathrm{ZO}$ & PS & PM & PB \\
\hline NB & NB & NB & NM & NM & NS & $\mathrm{ZO}$ & $\mathrm{ZO}$ \\
\hline NM & NB & NB & NM & NS & NS & $\mathrm{ZO}$ & $\mathrm{ZO}$ \\
\hline NS & NB & NM & NS & NS & $\mathrm{ZO}$ & PS & PS \\
\hline $\mathrm{ZO}$ & $\mathrm{NM}$ & NM & NS & $\mathrm{O}$ & PS & PM & PM \\
\hline PS & $\mathrm{NM}$ & NS & $\mathrm{ZO}$ & PS & PS & PM & PM \\
\hline PM & $\mathrm{ZO}$ & $\mathrm{ZO}$ & PS & PS & PM & PB & PB \\
\hline PB & $\mathrm{ZO}$ & $\mathrm{ZO}$ & PS & PM & PM & PB & PB \\
\hline \multicolumn{8}{|c|}{ Rule table of fuzzypid control of $\Delta \mathrm{kd}$} \\
\hline elec & NB & NM & NS & $\mathrm{ZO}$ & PS & PM & PB \\
\hline NB & PS & NS & NB & NB & NM & $\mathrm{NM}$ & PS \\
\hline NM & PS & NS & NB & NM & NM & NS & $\mathrm{ZO}$ \\
\hline NS & $\mathrm{ZO}$ & NS & $\mathrm{NM}$ & NM & NS & NS & $\mathrm{ZO}$ \\
\hline $\mathrm{ZO}$ & $\mathrm{ZO}$ & NS & NS & NS & NS & NS & $\mathrm{ZO}$ \\
\hline PS & $\mathrm{ZO}$ & $\mathrm{ZO}$ & $\mathrm{ZO}$ & $\mathrm{ZO}$ & $\mathrm{ZO}$ & $\mathrm{ZO}$ & $\mathrm{ZO}$ \\
\hline PM & PB & NS & PS & PS & PS & PS & PB \\
\hline
\end{tabular}

In view of the above simulation analysis: the fuzzy PID is more powerful than the classical PID. In this paper, the method is applied to the pitch attitude control of multi rotor UAV. When the multi rotor UAV is disturbed, for example, the load change, the center of gravity shift or the gust disturbance, it is used to improve the attitude stability control quality of the multi rotor UAV. I selected a commonly used in agricultural plant protection or eight rotor express transportation control model of the pitch channel of UAV as simulation object [5].

$$
\mathrm{G}_{\theta}(\mathrm{s})=\frac{303.3 \mathrm{~s}^{3}+344.4 \mathrm{~s}^{2}+34.56 \mathrm{~s}+0.8169}{\mathrm{~s}^{5}+7.29 \mathrm{~s}^{4}+7.101 \mathrm{~s}^{2}+3.744 \mathrm{~s}^{2}+3.364 \mathrm{~s}+0.2487}
$$

According to the load change of the eight rotor unmanned aerial vehicle (UAV), or the influence of the gust disturbance on the attitude control system. Such as plant protection machine liquid in the medicine box shaking caused by the body center of gravity of the cyclical changes on the attitude control system [7], the writer uses MATLAB to control pitch control channel simulation tracking curve, this paper assumes that the amplitude of sinusoidal interference to the superposition of 6 attitude control system simulation of the eight rotor UAV has received the response of periodic disturbance when the flight attitude control. It is shown in figure 5 and figure 6. 


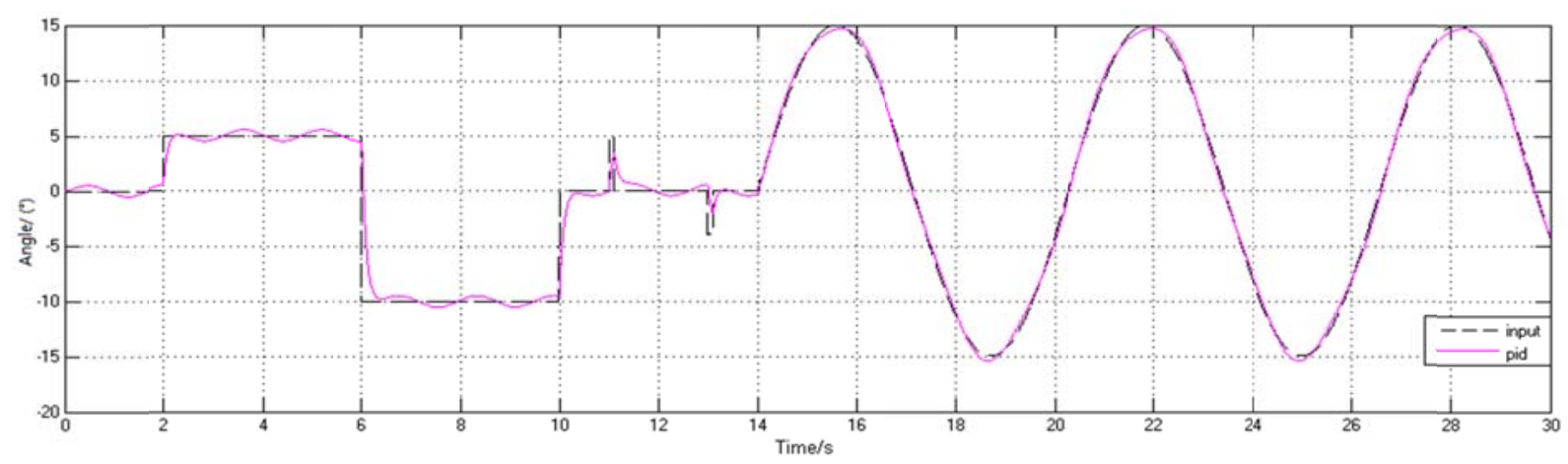

FIGURE. 5 Simulation tracking curve of the classic PID eight rotor pitch control amplitude under 6 strong interference.

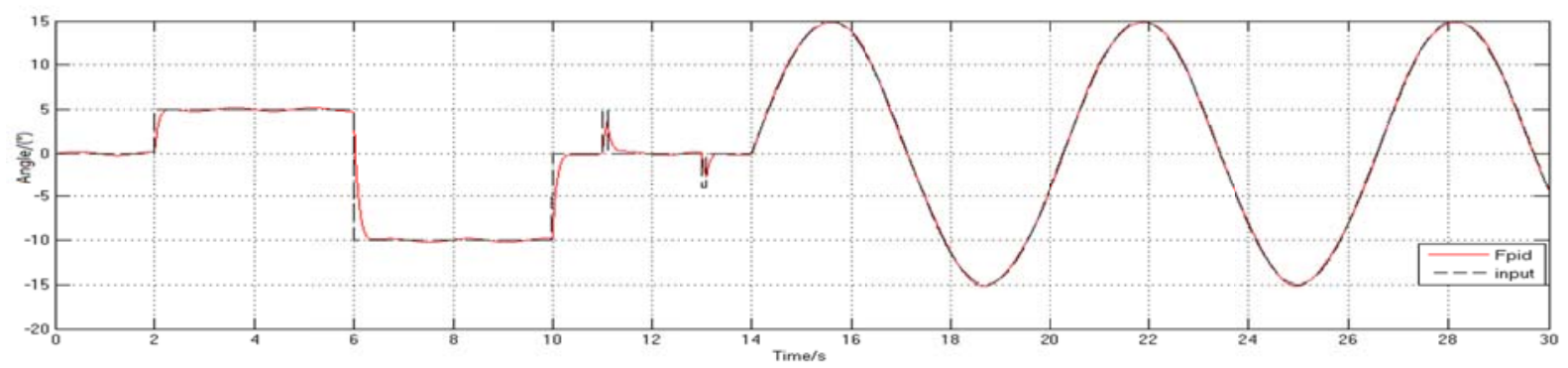

FIGURE. 6 Simulation tracking curve of Fuzzy PID eight rotor pitch control amplitude under 6 strong interference.

\section{CONCLUSION}

The simulation results show that the fuzzy PID control is better than the classical PID, and has good robustness. The two systems are close to zero. In the two indexes of steady state error and overshoot, the control quality of fuzzy PID is higher. The application of fuzzy PID to control the eight rotor UAV pitch channel can achieve accurate control output under strong disturbance.

\section{ACKNOWLEDGMENTS}

This work was financially supported by Mr. Qi Wang fund.

Corresponding author is Qi Wang. Email: 641617562@qq.com

\section{REFERENCES}

[1] Wang Shuyan, Shi Yu, Feng Zhongxu. Research on control method based on fuzzy PID controller [J]. mechanical science and technology. 2011,30 (01): 166-172.

[2] Wang Lixin. Fuzzy system and Fuzzy Control Tutorial [M]. Tsinghua University press.2003.

[3] Yin Yunhua, Shuikang fan, Chen Mine. Adaptive fuzzy PID controller design and Simulation of [J]. fire control and command. 2008,33 (07): 96-99

[4] De Xiang Yi. The fuzzy PID parameter prediction model based on self-tuning control algorithm and Realization of [D]. 2008 Northeastern University.

[5] Li Weirong, Fang, Li Chenglong. Modeling and control of unmanned aerial vehicle (UAV) with suspended load eight [J]..2015,35 application (S2): 138-142.

[6] Zhao Yan. Research on fuzzy PID control method based on fuzzy theory and conventional PID control [J]. Shandong electric power technology, 2009 (6): 54-56,63.

[7] Liu Haopeng, the dragon river, Wan Peng. Fuzzy PID control [J]. Journal of agricultural engineering plant protection four axis aircraft. 2015,31 (1): 71-77. 